\title{
Duration of dentin tubule occlusion by the calcium phosphate precipitation method: An in vivo study in beagle dogs
}

\author{
Toshiyuki SUGE ${ }^{1}$, Kunio ISHIKAWA², Takashi MATSUO ${ }^{1}$ and Shigeyuki EBISU³ \\ ${ }^{1}$ Department of Conservative Dentistry, Institute of Biomedical Sciences, Tokushima University Graduate School, 3-18-15 Kuramoto, Tokushima \\ 770-8504, Japan \\ ${ }^{2}$ Department of Biomaterials, Faculty of Dental Science, Kyushu University, 3-1-1 Maidashi, Higashi-ku, Fukuoka 812-8582, Japan \\ ${ }^{3}$ Department of Restorative Dentistry and Endodontology, Division of Oral Infections and Disease Control, Osaka University Graduate School of \\ Dentistry, 1-8 Yamadaoka, Suita, Osaka 565-0871, Japan \\ Corresponding author, Toshiyuki SUGE; E-mail: suge@tokushima-u.ac.jp
}

\begin{abstract}
The aim of the present study was to assess the duration of dentin tubule occlusion by the calcium phosphate precipitation (CPP) method in the vital teeth of beagle dogs. Vital teeth were treated using the CPP method, potassium oxalate, or a bonding agent (Liner bond II) after cavity preparation and acid etching. The dentin tubules of all groups, except for the bonding agent, opened more widely with time in the absence of plaque control. Dentin tubules treated with the CPP method were open and no precipitate remained in the absence of plaque control. Differences were observed in dentin tubule occlusion when plaque control was achieved by daily tooth brushing. The majority of dentin tubules were occluded with an apatitic precipitate seven days after the CPP method with plaque control. The present results demonstrated that the CPP method is useful with proper plaque control.
\end{abstract}

Keywords: Hydroxyapatites, Calcium phosphates, Dentin tubules, Occlusion, Dentin hypersensitivity

\section{INTRODUCTION}

The calcium phosphate precipitation (CPP) method was developed to treat dentin hypersensitivity and the treatment solutions used have been improved for clinical applications ${ }^{1-4)}$. The improved CPP method involves the sequential application of two solutions. A CPP solution ( $1 \mathrm{~mol} / \mathrm{L} \mathrm{CaHPO}{ }_{4} \cdot 2 \mathrm{H}_{2} \mathrm{O}$ dissolved into $2 \mathrm{~mol} / \mathrm{L} \mathrm{H}_{3} \mathrm{PO}_{4}$ ) is initially applied to the surface of dentin and penetrates dentin tubules by capillary action. This is followed by the application of a post-treatment solution $(1 \mathrm{~mol} / \mathrm{L}$ $\mathrm{NaHCO}_{3}$ with $0.3 \mathrm{~mol} / \mathrm{L} \mathrm{NaF}$ ) to neutralize the CPP solution. Immediately after the application of the posttreatment solution, dentin tubules are occluded with apatitic mineral. The depth of occlusion with the CPP method was previously shown to be approximately 10 $\mu \mathrm{m}$ in an in vitro study using dentin disks made from extracted human teeth. However, the depth of occlusion was reduced by $50 \%$ when the CPP method was applied to the vital teeth of beagle dogs, even though dentin tubules were homogeneously and completely occluded with apatitic mineral, as described in the in vitro study ${ }^{5}$. Reductions in the depth of precipitation in the in vivo study were attributed to the presence of dentin fluid and pulpal pressure towards the exterior in vital teeth. Pulpal pressure was previously reported to be 14.1 $\mathrm{cmH}_{2} \mathrm{O}$ from the pulpal side toward the exterior ${ }^{6)}$, and prevents the penetration of substances, such as resin monomers, through dentin tubules ${ }^{7}$. The reductions achieved in the depth of occlusion by the CPP method may also affect the duration of dentin tubule occlusion.

Regarding the durability of the CPP method, our previous in vitro findings indicated that the apatitic precipitate formed by the CPP method was stable and insoluble in synthetic saliva ${ }^{8}$. Since saliva is supersaturated with hydroxyapatite (HAP) $)^{9,10)}$, the addition of more calcium phosphate results in precipitation after the dissolution of an initially small amount of the precipitate. The factors contributing to precipitation in dentin tubules have been reported to differ under in vitro and in vivo conditions. Plaque, beverages, food, and daily brushing have been shown to influence the duration of dentin tubule occlusion in vivo. However, the accumulation of plaque on the surface of dentin was not assessed in the in vitro study. Plaque has been shown to play an important role in the appearance and aggravation of dentin hypersensitivity ${ }^{11,12)}$.

The aim of the present study was to evaluate the stability of the CPP method with or without plaque control using the vital teeth of beagle dogs. The occlusion ability of the CPP method was compared with those of potassium oxalate and a bonding agent, which are typically used to treat dentin hypersensitivity.

\section{MATERIALS AND METHODS}

\section{Preparation of treatment solutions}

The CPP solution was prepared as described previously ${ }^{11}$. In brief, $1 \mathrm{~mol} / \mathrm{L}$ of reagent grade dicalcium phosphate dihydrate (DCPD: Nacalai Tesque, Kyoto, Japan) was dissolved in $2 \mathrm{~mol} / \mathrm{L} \mathrm{H}_{3} \mathrm{PO}_{4}$ solution at room temperature to prepare DCPD-saturated CPP solution $(\mathrm{pH}=1.4)$. Clear solutions $\left([\mathrm{Ca}]=1 \mathrm{~mol} / \mathrm{L} ;\left[\mathrm{PO}_{4}\right]=3 \mathrm{~mol} / \mathrm{L}\right)$ were filtered through $0.22-\mu \mathrm{m}$ Millex-GS filter assemblies (Millipore, Bedford, MA, USA). Post-treatment solution was also prepared from reagent grade chemicals as described previously ${ }^{4)}$. The post-treatment solution was a $1 \mathrm{~mol} / \mathrm{L} \mathrm{NaHCO}_{3}$ aqueous solution containing $0.3 \mathrm{~mol} / \mathrm{L}$ 
$\mathrm{NaF}$ ( $\mathrm{pH}=8.3$ ). Twenty-five weight percent dipotassium oxalate solution $(\mathrm{pH}=8.8)$ was prepared from reagent grade chemicals (Nacalai Tesque) for comparisons. The bonding agent Liner Bond II (Kuraray, Tokyo, Japan) was used as a control treatment.

\section{Treatment of vital teeth with the CPP method, potassium} oxalate, or resin liner

The use of dogs for this experiment conformed to the guidelines of the Committee on Animal Care and the Use of Laboratory Animals at Tokushima University. Fifteen beagle dogs (Ridglan Research Farms, Mt. Horeb, WI, USA) with sound teeth and weighing between 10 and $12 \mathrm{~kg}$ were used in the present study. Five dogs were assigned to each treatment group. Experiments were performed on dogs as described in our previous study ${ }^{5,12}$. Dogs were anesthetized with an intravenous injection of $5 \%$ sodium pentobarbital $(20 \mathrm{mg} / \mathrm{kg}$ body weight, nembutal; Dainabot, Osaka, Japan). Class V cavities were prepared on the buccal surfaces of the maxillary and mandibular molars and canines using a sharp diamond point with a high-speed handpiece under water cooling. Each cavity was prepared by removing enamel to a depth of approximately $1 \mathrm{~mm}$ from the enamel surface. Cavities were prepared without local anesthesia to avoid changes in pulpal pressure ${ }^{13)}$. The tooth was then etched with $50 \%$ citric acid for $2 \mathrm{~min}$ to remove the smear layer and open dentin tubules; this procedure simulated patent dentin tubules that are typical of hypersensitive dentin ${ }^{14)}$. The tooth was then washed with distilled water and dried with a three-way syringe. This specimen served as a control. In CPP method-treated teeth, the CPP solution was applied to dentin with a cotton swab for $1 \mathrm{~min}$, followed by gentle drying with a cotton swab. The post-treatment solution was then applied to dentin with a cotton swab for $1 \mathrm{~min}$. These procedures were repeated twice to increase the depth of occlusion of the precipitate in dentin tubules. Similarly, 25\% potassium oxalate solution was applied to exposed dentin with a cotton swab for $3 \mathrm{~min}$. In the case of the bonding agent, Liner Bond II was applied to teeth with a sponge and then light-cured for $30 \mathrm{~s}$ according to the manufacturer's instructions without etching. After the treatment with the CPP method, potassium oxalate, or resin liner, the tooth was washed with distilled water.

Throughout the experimental period, beagle dogs were provided daily with approximately $300 \mathrm{~g}$ of a commercial ration (CD-5, Crea, Tokyo, Japan) and tap water.

\section{Plaque control}

Plaque control was achieved by brushing without toothpaste for several minutes every day after meals on left side teeth only. An electric brush (Dent EX, Morita, Tokyo, Japan) was used for plaque control with approximately $250 \mathrm{~g}$ of pressure until plaque was completely removed according to an oral inspection. Nonplaque control teeth (right side teeth) were not subjected to brushing throughout the experimental period.

\section{Dentin biopsy}

Dentin biopsy was performed under intravenous anesthesia. To obtain dentin specimens (with a diameter of approximately $0.8 \mathrm{~mm}$ and depth of $0.5-0.8 \mathrm{~mm}$ ), dentin biopsy was performed immediately and seven days after treatment with a cylindrical diamond point using a high-speed handpiece under water cooling ${ }^{5,12,15,16)}$. After recovery, the cavity was filled with glass-ionomer cement. Dentin biopsy specimens were rinsed with distilled water for $1 \mathrm{~min}$ and dried with a critical point dryer (Hitachi, Tokyo, Japan). In the plaque control group, ultrasonic cleaning was not performed due to plaque was removed by daily brushing. Specimens obtained from non-plaque control teeth were rinsed with distilled water and placed in an ultrasonic cleaner for 30 min to remove accumulated plaque at the dentin surface and dried using a critical point dryer.

\section{SEM observations}

The occlusion ability of the three treatment methods was assessed by observations under a scanning electron microscope (SEM; S-700, Hitachi). The specimens obtained by the above procedures were mounted on metal holders for gold coating, and the surface of biopsy specimens was observed with an accelerating voltage of $20 \mathrm{kV}$.

\section{Percentage of open dentin tubules}

The percentages of open dentin tubules with the three treatment methods (the CPP method, potassium oxalate, and the bonding agent) were quantitatively assessed using computer image analysis software with SEM photographs. After SEM observations, SEM photographs (original magnification $\times 4,000$ ) were scanned into a computer using a scanner, and the area of open dentin tubules was marked using a computer program (Adobe Photoshop, Adobe ver 4.0.1 for Power PC, Tokyo, Japan). Imaging software (NIH image ver 1.62) was used to measure the area of open dentin tubules per unit area. Adherent bacteria were excluded from the calculation of the percentage of open dentin tubules. The percentage of open tubules per unit area was defined and calculated as an index of dentin tubule occlusion, as shown in the following equation: the percentage of open tubules $(\%)=($ area of open dentin tubules/unit area $) \times 100$. A total of 16 biopsy specimens, including the plaque control groups (8 specimens) and non-plaque control groups ( 8 specimens) were collected from one dog. Ten different images were randomly photographed so that the observation sites did not overlap on each biopsy specimen. The percentage of open dentin tubules in each treatment group was calculated from 400 SEM photographs ( 5 dogs $\times 8$ biopsy specimens $\times 10$ different images).

\section{Statistical analysis}

In statistical analyses, nonparametric Kruskal-Wallis test used as a post-hoc test, were performed using a statistical program (SPSS, Chicago, IL). $p$-Values $<0.05$ were considered to indicate significant differences. 


\section{RESULTS}

Figures 1 to 7 show typical SEM pictures of biopsy specimens taken from the vital teeth of beagle dogs immediately and seven days after treatment. Figure 1 shows typical SEM images of a biopsy specimen taken immediately after etching with 50\% citric acid for 2 min. Dentin tubules were open in a similar manner to hypersensitive dentin. Figure 2 shows typical SEM images taken immediately after the CPP method (Fig. 2a), potassium oxalate (Fig. $2 \mathrm{~b}$ ), and the bonding agent (Fig. 2c). Dentin tubules were occluded after the CPP method and bonding agent; however, some remained open immediately after the treatment with potassium oxalate, although calcium oxalate crystals had attached to peri- and inter-tubular dentin. Typical SEM images of control specimens in the plaque control group taken after seven days are shown in Fig. 3. Some dentin tubules had a slightly smaller diameter as a consequence of remineralization with plaque control. Figure 4 shows typical SEM images seven days after the CPP method with plaque control. Some dentin tubules opened with dissolution of the precipitate, and open dentin tubules were mixed with occluded dentin tubules. Figure 5 shows typical SEM images seven days after the potassium oxalate treatment with plaque control. Although the majority of dentin tubules were open, the diameter of open tubules decreased with the adherence of a spherical-

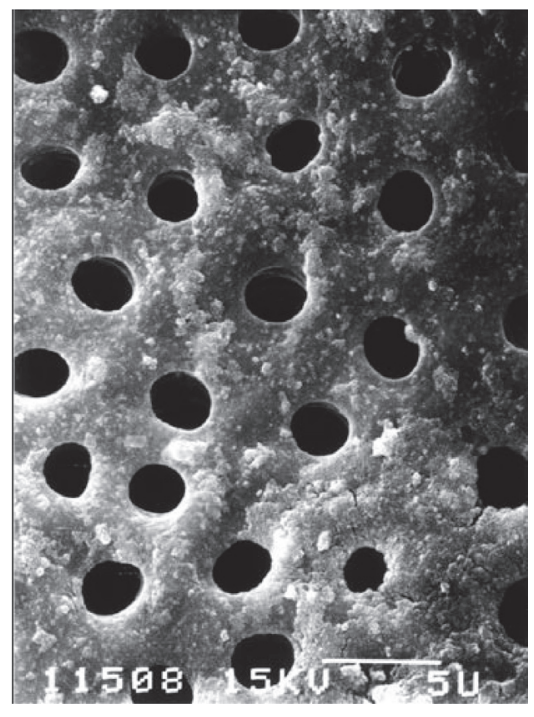

Fig. 1 Typical scanning electron micrographs of the surface of dentin biopsy specimens obtained immediately after etching with $50 \%$ citric acid for 2 min (Control specimen). The bar represents $5 \mu \mathrm{m}$.

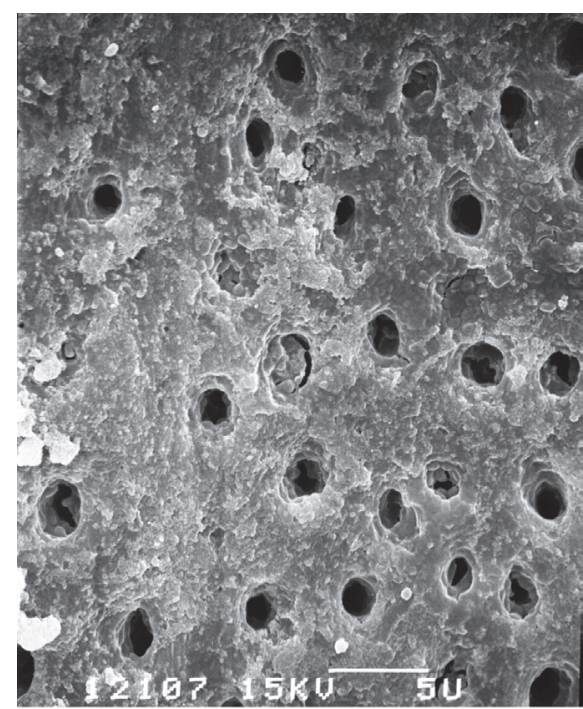

Fig. 3 Typical scanning electron micrographs of the surface of dentin biopsy control (non-treatment) specimens obtained after seven days from the plaque control group. The bar represents $5 \mu \mathrm{m}$.
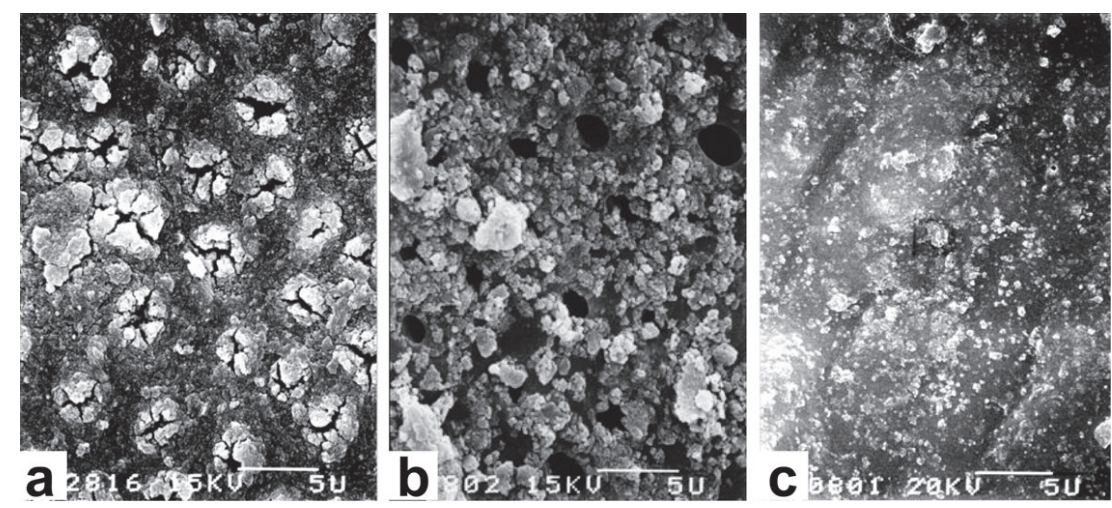

Fig. 2 Typical scanning electron micrographs of the surface of dentin biopsy specimens prepared from the vital tooth of a dog: (a) immediately after the CPP method; (b) Immediately after the $25 \%$ potassium oxalate treatment; (c) immediately after the bonding agent (Liner bond II) treatment. The bar represents $5 \mu \mathrm{m}$. 

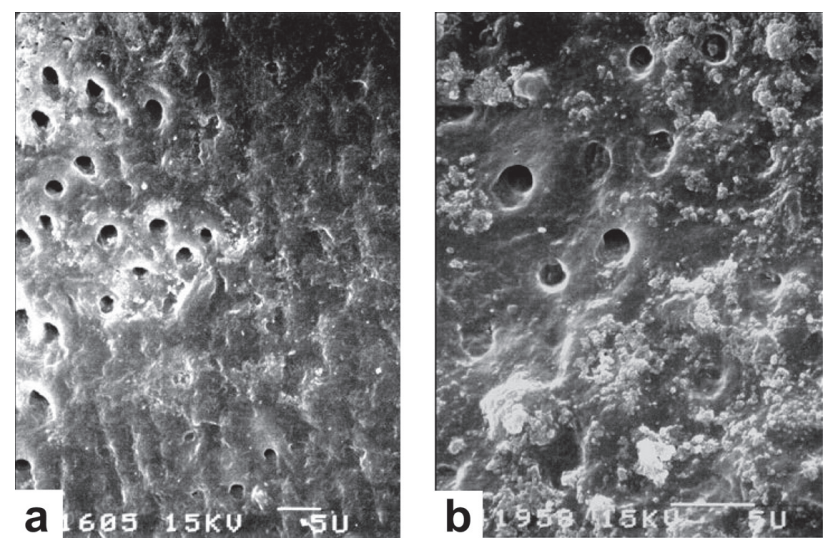

Fig. 4 Typical scanning electron micrographs of the surface of dentin biopsy specimens obtained seven days after the CPP method in the plaque control group: (a) low magnification; (b) high magnification. The bar represents $5 \mu \mathrm{m}$.
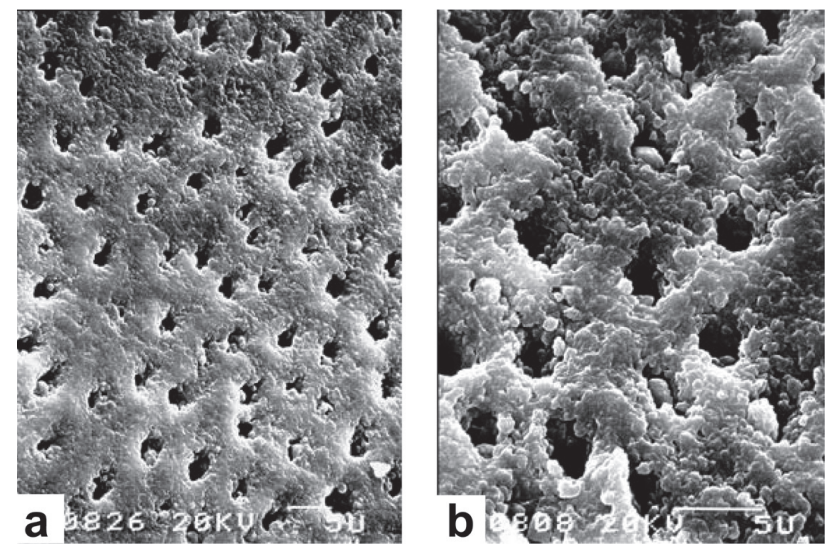

Fig. 5 Typical scanning electron micrographs of the surface of dentin biopsy specimens obtained seven days after the $25 \%$ potassium oxalate treatment in the plaque control group: (a) low magnification; (b) high magnification. The bar represents $5 \mu \mathrm{m}$. shaped precipitate. Figure 6 shows typical SEM images seven days after the bonding agent treatment with plaque control. The bonding agent still covered dentin tubules, similar to immediately after the treatment (Fig. $6 b)$. In some locations, the bonding agent was partially removed by brushing and the dentin surface appeared under the bonding agent (Fig. 6a). At these locations, the resin tag in dentin tubules was removed and patent dentin tubules were then observed.

In the non-plaque control group, the experimental results showed different aspects. In the control group (no treatment), the diameter of dentin tubules was wide with the demineralization of peri- and inter-tubular dentin (Fig. 7a). Furthermore, dentin tubules were open and no precipitate remained in dentin tubules seven days after the CPP method (Fig. 7b) and potassium oxalate treatment (Fig. 7c). In contrast, the bonding agent completely covered the surface of dentin, and dentin tubules were not observed after seven days
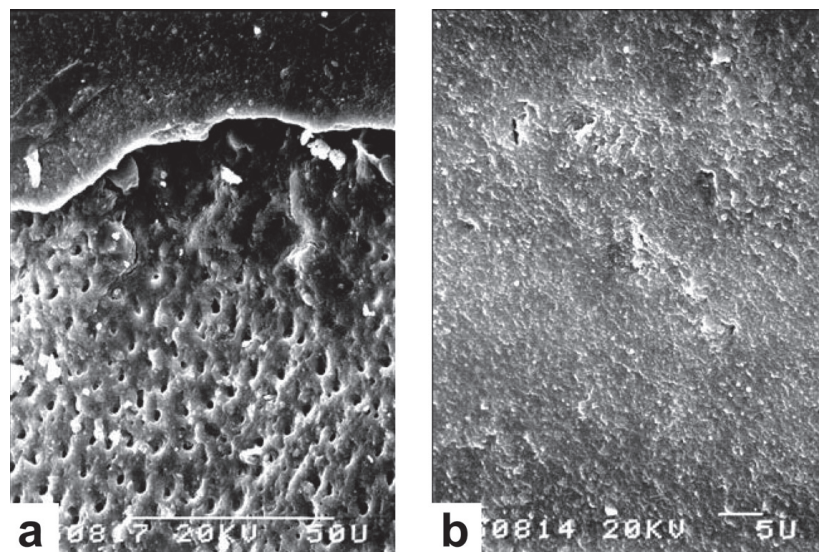

Fig. 6 Typical scanning electron micrographs of the surface of dentin biopsy specimens obtained seven days after the bonding agent (Liner bond II) treatment in the plaque control group: (a) low magnification. The bar represents $50 \mu \mathrm{m}$; (b) high magnification. The bar represents $5 \mu \mathrm{m}$.

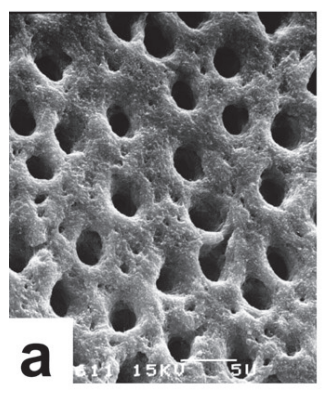

Control

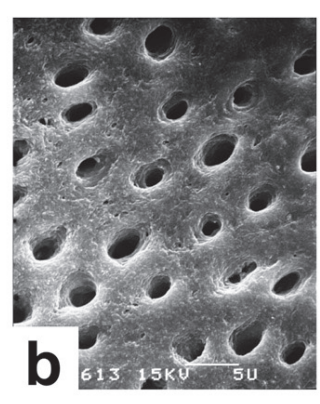

CPP

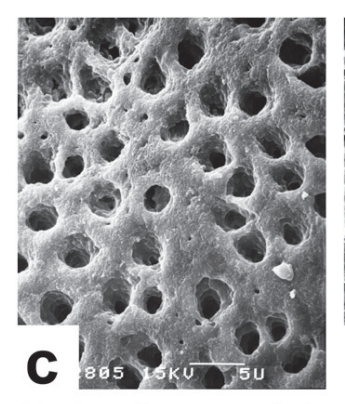

Potassium oxalate

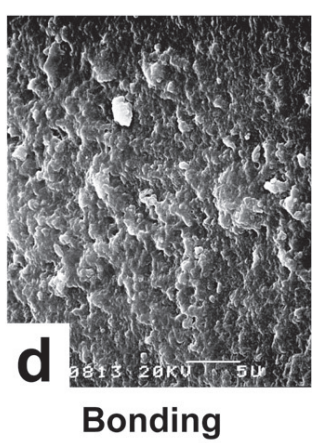

Fig. 7 Typical scanning electron micrographs of the surface of dentin biopsy specimens obtained seven days after the treatment from non-plaque control tooth: (a) control; (b) CPP method; (c) 25\% potassium oxalate treatment; (d) Bonding agent (Liner bond II) treatment. The bar represents $5 \mu \mathrm{m}$. 
Table 1 Percentage of open dentin tubules: Means \pm S.D

\begin{tabular}{lccc}
\hline \multicolumn{1}{c}{ Treatment } & CPP & Potassium oxalate & Bonding agent \\
\hline Before & $40.8 \pm 3.3$ & $40.8 \pm 3.3$ & $40.8 \pm 3.3$ \\
Immediately after treatment & $1.9 \pm 4.6^{*}$ & $36.7 \pm 3.9$ & $0.0 \pm 0.0^{*}$ \\
7 days after treatment (Plaque control) & $16.7 \pm 5.2^{*}$ & $23.3 \pm 4.3^{*}$ & $1.1 \pm 1.3^{*}$ \\
7 days after treatment (Non-plaque control) & $34.6 \pm 3.1$ & $38.2 \pm 2.8$ & $0.0 \pm 0.0^{*}$ \\
\hline
\end{tabular}

*A significant difference was observed between before and after treatment $(p<0.05)$.

$\mathrm{N}=400$ (5 dogs $\times 8$ biopsy specimens $\times 10$ different images).

(Fig. 7d).

Table 1 summarizes the percentage of open dentin tubules after the CPP method, potassium oxalate, and bonding agent in both the plaque control and nonplaque control groups. The occlusion ratio of all groups increased after seven days, except for the bonding agent group without plaque control. The occlusion ratio of the CPP method in both the plaque control and non-plaque control groups was inferior to that with the bonding agent seven days after treatment. However, the CPP method achieved better occlusion than potassium oxalate in both the plaque control and non-plaque control groups.

\section{DISCUSSION}

The CPP method achieved the continuous occlusion of dentin tubules when teeth were subjected to plaque control by brushing only. When plaque control was not performed after the CPP method, the apatitic precipitate did not remain in dentin tubules and dentin tubules opened due to demineralization of the dental plaque. The crystallinity of the apatitic precipitate formed by the CPP method was lower than that by tooth HAP; therefore, it was more easily demineralized by plaque than dentin. The CPP method increased the crystallinity of apatite and the acid resistance of enamel because the post-treatment solution in the CPP method contained fluoride ${ }^{17)}$. Therefore, the demineralization of peritubular dentin was prevented and the percentage of open dentin tubules with the CPP method in the non-plaque control group was slightly lower than those with the control and potassium oxalate treatments. Plaque control strongly influences the etiology of dentin hypersensitivity. The $\mathrm{pH}$ of plaque and plaque products is acidic ${ }^{18,19)}$, and, thus, induces the demineralization of dentin and open dentin tubules. The relationship between the conditions of plaque control and the patency and/or occlusion of dentin tubules using an intraoral model or in an in vivo study have been reported ${ }^{11,12)}$. The findings obtained showed that the diameters of dentin tubules increased with time in the non-plaque control group, which is consistent with the results of the present study. The open ratio of dentin tubules was approximately four-fold higher after three weeks than the initial value. On the other hand, in both plaque control groups, (Group 1): plaque control was mechanically achieved by daily brushing without toothpaste, and (Group 2): plaque control was chemically achieved using chlorhexidine, which slightly decreased the diameter of dentin tubules with time. Under these conditions, mineral deposits from saliva were observed in some dentin tubules. The component of the precipitate that formed in dentin tubules was identified as calcium phosphate by energy dispersive X-ray analyses ${ }^{11,12)}$. The deposition of calcium phosphate was also observed in plaque control specimens in the present study. Dentists generally agree that hypersensitive teeth will recovery with adequate plaque control. A previous study reported that open dentin tubules were occluded when proper non-toothpaste brushing was performed, even though the findings obtained were from an in vitro study ${ }^{20)}$. Some of the bacteria that attached to the dentin surface and tubule wall were observed in a few plaque control specimens; therefore, the use of antibacterial mouthwash is important for the complete removal of bacteria in combination with daily brushing. The attachment of plaque on the tooth surface may be suppressed by an antibacterial mouthwash, such as chlorhexidine, resulting in a decrease in or the prevention of tooth demineralization by plaque and plaque products. Sequential rinses with chlorhexidine and sodium fluoride have been shown to significantly reduce dentin hypersensitivity in a clinical study ${ }^{21)}$. Proper plaque control is also indispensable for the occlusion of dentin tubules for long periods in the CPP method. Consistent with the present results, a previous study reported that the occlusion of dentin tubules with an amorphous calcium phosphate was effective for the treatment of dentin hypersensitivity in a clinical study ${ }^{22}$.

The durability of occlusion with the CPP method was compared with that by potassium oxalate and a bonding agent in the present study. The occlusion ability of the potassium oxalate treatment was weaker than that of the CPP method both immediately and seven days after treatment. Potassium oxalate occludes open dentin tubules with calcium oxalate. The duration of dentin tubule occlusion was previously reported to be short lived; however, calcium oxalate is a relatively insoluble mineral ${ }^{23)}$. The occlusion ability of potassium oxalate was found to be markedly affected by the degree of demineralization of the tooth surface ${ }^{24)}$. Therefore, the amount of calcium oxalate that formed after the potassium oxalate treatment was reduced due to 
simulated hypersensitive teeth being prepared with $50 \%$ citric acid etching. In contrast, the bonding agent achieved complete occlusion and most of the dentin surface was still covered in both the plaque control and non-plaque control groups seven days after treatment. In a clinical study, bonding agents were shown to be effective in the treatment of dentin hypersensitivity ${ }^{25,26)}$. Since bonding agents are organic materials, demineralization by dental plaque and an acid may be difficult. In the non-plaque control group, the bonding agent still completely covered the dentin surface after seven days. The percentage of open dentin tubules with the bonding agent was higher in the nonplaque control group than in the plaque control group. The exfoliation of the bonding agent may have due to the mechanical force of brushing. Therefore, bonding agents are recommended for patients unable to perform proper plaque control due to tactile pain. On the other hand, inorganic minerals, i.e. calcium phosphate and calcium oxalate, more strongly influence chemical factors, such as plaque accumulation and acidic beverages, than mechanical factors, including daily brushing.

In the present study, SEM observations were limited to the dentin surface; however, potassium oxalate solution penetrates deeply into dentin tubules, even in vital dog teeth ${ }^{5,27)}$. Therefore, dentin surface morphology is not always represented in clinical symptoms. If dentin tubules are blocked with a precipitate at a high depth, patients have no symptoms of hypersensitive teeth even though dentin tubules are open at the dentin surface. The present results indicate that further in vivo evaluations are needed before the clinical application of the CPP method.

\section{CONCLUSION}

The CPP method has potential in the treatment of dentin hypersensitivity. However, its effects may be diminished in patients who do not perform proper plaque control.

Organic dental materials, such as resin bonding or similar materials, are more strongly recommended for patients with poor plaque control than inorganic dental materials (calcium phosphate and potassium oxalate).

\section{ACKNOWLEDGMENTS}

The authors declare no conflicts of interest. This study was supported in part by Grants-in-Aid for Scientific Research (09557152 and 16591916) from the Ministry of Education, Culture, Sports, Science and Technology in Japan.

\section{REFERENCES}

1) Ishikawa K, Suge T, Yoshiyama M, Kawasaki A, Asaoka K, Ebisu S. Occlusion of dentinal tubules with calcium phosphate using acidic calcium phosphate solution followed by neutralization. J Dent Res 1994; 73: 1197-1204.

2) Suge T, Ishikawa K, Kawasaki A, Yoshiyama M, Asaoka K, Ebisu S. Effects of fluoride on the calcium phosphate precipitation method for dentinal tubule occlusion. J Dent
Res 1995; 74: 1079-1085.

3) Kawasaki A, Ishikawa K, Suge T, Yoshiyama M, Asaoka K, Ebisu S. Effects of hexafluorosilicate on the precipitate composition and dentine tubule occlusion by calcium phosphate. J Dent 1996; 24: 429-434.

4) Suge T, Ishikawa K, Kawasaki A, Suzuki K, Matsuo T, Ebisu S. Evaluation of post-treatment solutions for clinical use with the calcium phosphate precipitation method. J Dent 1999; 27 : 487-496.

5) Suge T, Ishikawa K, Kawasaki A, Suzuki K, Matsuo T, Noiri $\mathrm{Y}$, et al. Calcium phosphate precipitation method for the treatment of dentin hypersensitivity. Am J Dent 2002; 15: 220-226.

6) Ciucchi B, Bouillaguet S, Holz J, Pashley D. Dentinal fluid dynamics in human teeth, in vivo. J Endod 1995; 21: 191-194.

7) Gerzina TM, Hume WR. Effect of hydrostatic pressure on the diffusion of monomers through dentin in vitro. J Dent Res 1995; 74: 369-373.

8) Suge T, Ishikawa K, Kawasaki A, Yoshiyama M, Asaoka K, Ebisu S. Duration of dentinal tubules occlusion formed by calcium phosphate precipitation method: in vitro evaluation using synthetic saliva. J Dent Res 1995; 74: 1709-1714.

9) Rathje W. Oversaturation of saliva with hydroxyapatite. J Dent Res 1956; 35: 245-248.

10) Hay DI, Schluckebier SK, Moreno EC. Equilibrium dialysis and ultrafiltration studies of calcium and phosphate binding by human salivary proteins. Implication for salivary supersaturation with respect to calcium phosphate salts. Calcif Tissue Int 1982; 34: 531-538.

11) Kawasaki A, Ishikawa K, Suge T, Shimizu H, Suzuki K, Matsuo T, et al. Effects of plaque control on the patency and occlusion of dentine tubules in situ. J Oral Rehabil 2001; 28: 439-449.

12) Suge T, Kawasaki A, Ishikawa K, Matsuo T, Ebisu S. Effects of plaque control on the patency of dentinal tubules: an in vivo study in beagle dogs. J Periodontol 2006; 77: 454-459.

13) Kim S, Edwall L, Trowbridge H, Chein S. Effects of local anesthetics on pulpal blood flow in dogs. J Dent Res 1984; 63: 650-652.

14) Greenhill JD, Pashley DH. The effects of desensitizing agents on the hydraulic conductance of human dentin in vitro. $\mathrm{J}$ Dent Res 1981; 60: 686-698.

15) Yoshiyama M, Masada J, Uchida A, Ishida H. Scanning electron microscopic characterization of sensitive vs. insensitive human radicular dentin. J Dent Res 1989; 68: 1498-1502.

16) Yoshiyama M, Noiri Y, Ozaki K, Uchida A, Ishikawa Y, Ishida H. Transmission electron microscopic characterization of hypersensitive human radicular dentin. J Dent Res 1990; 69: 1293-1297.

17) Suge T, Kawasaki A, Ishikawa K, Matsuo T, Ebisu S. Effects of calcium phosphate precipitation method on acid resistance to apatite powder and bovine tooth. Dent Mater J 2008; 27: 508-514.

18) Kleinberg I, Jenkins GN. The $\mathrm{pH}$ of dental plaques in the different areas of the mouth before and after meals and their relationship to the $\mathrm{pH}$ and rate of flow of resting saliva. Arch Oral Biol 1964; 9: 493-516.

19) Tanaka J, Mukai N, Tanaka M, Tanaka M. Relationship between cariogenic bacteria and $\mathrm{pH}$ of dental plaque at margin of fixed prostheses. Int J Dent 2012; 2012: 452108. Doi: 10.1155/2012/452108. Epub 2012 Jan 11.

20) Kuroiwa M, Kodaka T, Kuroiwa M, Abe M. Dentine hypersensitivity. Occlusion of dentinal tubules by brushing with and without an abrasive dentifrice. J Periodontol 1994; 65: 291-296.

21) Lawson K, Gross KB, Overman PR, Anderson D. Effectiveness of chlorhexidine and sodium fluoride in reducing dentin hypersensitivity. J Dent Hyg 1991; 65: 340-344. 
22) Geiger S, Matalon S, Blasbalg J, Tung M, Eichmiller FC. The clinical effect of amorphous calcium phosphate (ACP) on root surface hypersensitivity. Oper Dent 2003; 28: 496-500.

23) Kerns DG, Scheidt MJ, Pashley DH, Horner JA, Strong SL, Van Dyke TE. Dentinal tubule occlusion and root hypersensitivity. J Periodontol 1991; 62: 421-428.

24) Suge T, Kawasaki A, Ishikawa $K$, Matsuo T, Ebisu S. Comparison of the occluding ability of dentinal tubules with different morphology between calcium phosphate precipitation method and potassium oxalate treatment. Dent
Mater J 2005; 24: 522-529.

25) Prati C, Cervellati F, Sanasi V, Montebugnoli L. Treatment of cervical dentin hypersensitivity with resin adhesives: 4-week evaluation. Am J Dent 2001; 14: 378-382.

26) Kakaboura A, Rahiotis C, Thomaidis S, Doukoudakis S. Clinical effectiveness of two agents on the treatment of tooth cervical hypersensitivity. Am J Dent 2005; 18: 291-295.

27) Ikora S, Närhi M, Kontturi-Närhi V, Yamamoto $H$. Precipitation of desensitizing agents in dog dentinal tubules in vivo. J Dent Res 1998; 77: 672 (Abstr 321). 